\title{
Auslander bounds and homological conjectures
}

Jiaqun Wei

\begin{abstract}
Inspired by recent works on rings satisfying Auslander's conjecture, we study invariants, called Auslander bounds, and prove that they have strong relations to some homological conjectures.
\end{abstract}

\section{Introduction}

Throughout this paper, rings are associative with nonzero identities and modules are left modules unless otherwise specified. Let $R$ be a ring, we denote by $\operatorname{Mod} R$ the category of all left $R$-modules, and we denote by $\bmod R$ the full subcategory of all $R$-modules having finitely generated projective resolutions.

Auslander posed the following conjecture (cf. [8]).

Auslander Conjecture: Let $R$ be an artin algebra. Then for every $M \in$ $\bmod R$, there exists an integer $b_{M}$ such that $\operatorname{Ext}_{R}^{i}(M, N)=0$ for all $i>b_{M}$ and for all $R$-modules $N \in \bmod R$ satisfying $\operatorname{Ext}_{R}^{j}(M, N)=0$ for all suffciently large $j$.

It is known that if the Auslander conjecture holds for all finite dimensional algebras then the finitistic dimension conjecture is true for all finite dimensional algebras [8]. However, the Auslander conjecture fails in general by counterexamples in $[11,17]$. Rings satisfying the assertion in the Auslander conjecture are studied in $[4,9,16]$. In [4] the authors investigate in detail the relationship between such rings and some homological conjectures, for instance, the Auslander-Reiten conjecture, which we recall as follows.

Auslander-Reiten Conjecture: Let $R$ be a ring. If $M \in \bmod R$ and $\operatorname{Ext}_{R}^{i}(M, M \oplus R)=0$ for all $i>0$, then $M$ is projective. 
In this paper, we continue the study and focus on the number $b_{M}$, which we called the left Auslander bound of $M$ and denote by $\mathrm{lAb} M$, for the fixed module $M$. Note that the Auslander bound can be $\infty$. We prove the following result.

Theorem. If $M \in \bmod R$ and $\operatorname{Ext}_{R}^{i}(M, M \oplus R)=0$ for all sufficiently large $i$, then $\mathrm{lAb} M$ coincides with the projective dimension of $M$.

The result has clear relation with the Auslander-Reiten conjecture. More generally, it relates Auslander bounds with an equivalent version of the Wakamatsu-tilting conjecture (EWTC for short, see Section 3 for details), which asserts that an $R$-module $T \in \bmod R$ is tilting if and only if

(1) $\operatorname{Ext}_{R}^{i}(T, T)=0$ for all $i>0$ and

(2) there is an exact sequence $0 \rightarrow R \rightarrow T_{0} \rightarrow \cdots \rightarrow T_{n} \rightarrow 0$ for some $n$, where each $T_{i} \in \operatorname{add} T$.

We refer to [1] for the history and development of tilting theory. In fact, we obtain that if $R$ satisfies the condition that $1 \mathrm{Ab} M<\infty$ for every $M \in \bmod R$, then $R$ satisfies the conjecture (EWTC). This extends [4, Theorem A]. As we see, all rings with finite global repetition index, in particular, the finite dimensional algebra $\mathcal{O} / \pi \mathcal{O}$, where $\mathcal{O}$ is a classical order of finite global dimension over a discrete valuation ring $D$ with uniformizing parameter $\pi$ and residue class field $K$, satisfies the condition in the last result.

The above theorem is proved in Section 2, after some investigations on basic properties of Auslander bounds. Relations between homological conjectures and Auslander bounds are presented in Section 3, where we also formulate some new homological conjectures.

We introduce some notions in the following.

Let $R$ be a ring and $C, D \in \operatorname{Mod} R$. Let $t$ be a non-negative integer. By $\operatorname{Ext}_{R}^{>t}(C, D)=0$ we mean that $\operatorname{Ext}_{R}^{i}(C, D)=0$ for all $i>t$. By $\operatorname{Ext}_{R}^{\gg}(C, D)=0$ we mean that $\operatorname{Ext}_{R}^{>t}(C, D)=0$ for some $t$. Given an $R$-module $M$ and an integer $t \geq 0$, we denote by $M^{>t}\left({ }^{>t} M\right.$, resp.) the subcategory of all modules $N$ such that $\operatorname{Ext}_{R}^{>t}(M, N)=0\left(\operatorname{Ext}_{R}^{>t}(N, M)=0\right.$, resp.). The notions $M \gg$ and $\gg M$ are defined similarly.

For an $R$-module $M$, we denote by add $M$ the class of all modules isomorphic to direct summands of finite direct sums of copies of $T$. We use $\operatorname{pd} M$ (id $M, \mathrm{fd} M$, resp.) to denote projective (injective, flat, resp.) dimension of $M$.

We denote by $R^{o}$ the opposite ring of $R$. Thus $\operatorname{Mod} R^{o}$ is the category of all right $R$-modules. In case that $R$ is an artin algebra, we denote by $\mathbf{D}$ the usual dual functor between $\bmod R$ and $\bmod R^{\circ}$. 


\section{Auslander bounds}

Throughout this section, we fix $R$ a ring. If a class $\mathcal{C}$ consists of a single $R$-module, say $C$, then we use $C$ instead of $\mathcal{C}$.

We introduce the following notion.

Definition 2.1 Let $\mathcal{C}$ and $\mathcal{D}$ be two classes of $R$-modules. The Auslander bound of the pair $(\mathcal{C}, \mathcal{D})$, denoted by $\operatorname{Ab}(\mathcal{C}, \mathcal{D})$, is defined in the following way:

- If there are no $C \in \mathcal{C}$ and $D \in \mathcal{D}$ such that $\operatorname{Ext}_{R}^{\gg}(C, D)=0$, then $\operatorname{Ab}(\mathcal{C}, \mathcal{D})=-1$

- Otherwise, $\operatorname{Ab}(\mathcal{C}, \mathcal{D})$ is the minimal non-negative integer $m$ such that $\operatorname{Ext}_{R}^{>m}(C, D)=0$ for any $C \in \mathcal{C}$ and $D \in \mathcal{D}$ with $\operatorname{Ext}_{R}^{\gg}(C, D)=0$, or $\infty$ if no such minimal integer exists.

It is easy to see that $\operatorname{Ab}(C, D)<\infty$ for any two $R$-modules $C, D$.

Let $M \in \operatorname{Mod} R$. It is also easy to see that $\operatorname{Ab}(M, \mathcal{C})$ is just the minimal non-negative integer $m$ such that $M^{\gg} \cap \mathcal{C}=M^{>m} \cap \mathcal{C}$, or $\infty$ if no such integer exists, or -1 if $M^{\gg} \bigcap \mathcal{C}=\varnothing$. Similarly $\operatorname{Ab}(\mathcal{C}, M)$ is just the minimal non-negative integer $m$ such that $\mathcal{C} \bigcap^{\gg} M=\mathcal{C} \bigcap^{>m} M$, or $\infty$ if no such integer exists, or -1 if $\mathcal{C} \cap \bigcap^{\gg}=\varnothing$.

We use the following simple notions for $M \in \operatorname{Mod} R$.

- $\operatorname{LAb} M:=\operatorname{Ab}(M, \operatorname{Mod} R)$ (called the big left Auslander bound of $M$ );

- $\operatorname{lAb} M:=\operatorname{Ab}(M, \bmod R)$ (the small left Auslander bound of $M)$;

- $\operatorname{RAb} M:=\operatorname{Ab}(\operatorname{Mod} R, M)$ (the big right Auslander bound of $M)$;

- $\operatorname{rAb} M:=\operatorname{Ab}(\bmod R, M)$ (the small right Auslander bound of $M)$.

It is easy to see that the above Auslander bounds are non-negative.

Remark 2.2 (1) LAbM is just the minimal bound on the vanishing of $\operatorname{Ext}_{R}(M,-)$ in [5]. In $[9,16], 1 \mathrm{Ab} M$ is also denoted by $e_{R}(M,-)$ in case $M \in \bmod R$.

(2) One can similarly define the Tor-Auslander bound of a pair $(\mathcal{C}, \mathcal{D})$, denoted by $\operatorname{tAb}(\mathcal{C}, \mathcal{D})$, by the bifunctor $\operatorname{Tor}_{i>m}^{R}(-,-)$. Then $\operatorname{tAb}(M, \operatorname{Mod} R)$ for a right $R$-module $M$ is just the minimal bound on the vanishing of $\operatorname{Tor}^{R}(M,-)$ in [13].

(3) Obviously, $\operatorname{LAb} M \leq \operatorname{pd} M$ and $\operatorname{RAb} M \leq \operatorname{id} M$ with the equality holds if the latter is finite. If $M \in \bmod R$, then $\operatorname{lAb} M=\operatorname{pd} M$ provided that $\operatorname{pd} M<\infty$. 
We say that $\mathcal{C}$ has the two-out-of-three property provided that any two terms in a short exact sequence are in $\mathcal{C}$ implies the third term is also in $\mathcal{C}$. The proof of the following lemma is easy.

Lemma 2.3 Let $M \in \operatorname{Mod} R$.

(1) All subcategories $\bmod R, M^{\gg}$, and ${ }^{\gg} M$ have the two-out-of-three property.

(2) All subcategories $\bmod R, M^{\gg}$, and ${ }^{\gg} M$ are closed under direct summands and finite direct sums. Moreover, $M^{\gg}\left({ }^{\gg} M\right.$, resp. $)$ is closed under arbitrary direct products (direct sums, resp.).

(3) $M^{\gg}=\left(\Omega^{i} M\right)^{\gg}$, where $\Omega^{i} M$ denotes an $i$-th syzygy of $M$.

(4) ${ }^{\gg} M=\gg\left(\Omega^{-i} M\right)$, where $\Omega^{-i} M$ denotes an $i$-th cosyzygy of $M$.

(5) If $R$ is an artin algebra and $M \in \bmod R$, then $\mathbf{D}\left(M^{\gg}\right)=\gg(\mathbf{D}(M))$.

Lemma 2.4 Let $M, N \in \operatorname{Mod} R$ and $\mathcal{C}$ be a class of $R$-modules.

(1) $\operatorname{Ab}(M \oplus N, \mathcal{C}) \leq \max \{\operatorname{Ab}(M, \mathcal{C}), \operatorname{Ab}(N, \mathcal{C})\}$.

(2) $\operatorname{rAb}(\mathcal{C}, M \oplus N) \leq \max \{\operatorname{Ab}(\mathcal{C}, M), \operatorname{Ab}(\mathcal{C}, N)\}$.

(3) If $R$ is an artin algebra and $M \in \bmod R$, then $\operatorname{lAb} M=\operatorname{rAb}(\mathbf{D}(M))$.

Proof. (1) Clearly we can assume that

$$
k:=\max \{\operatorname{Ab}(M, \mathcal{C}), \operatorname{Ab}(N, \mathcal{C})\}<\infty .
$$

Now note that

$$
(M \oplus N)^{\gg} \bigcap \mathcal{C}=M^{\gg} \bigcap N^{\gg} \bigcap \mathcal{C}=M^{>k} \bigcap N^{>k} \bigcap \mathcal{C}=(M \oplus N)^{>k} \bigcap \mathcal{C},
$$

so we have that $\operatorname{Ab}(M \oplus N, \mathcal{C}) \leq k$.

The proof of (2) is dual to that of (1). The proof of (3) follows from Lemma 2.3(5).

Proposition 2.5 Let $\mathcal{C}, \mathcal{D}, \mathcal{E}, \mathcal{F}$ be four classes of $R$-modules. If $\mathcal{C} \subseteq \mathcal{D}$ and $\mathcal{E} \subseteq \mathcal{F}$, then

$$
\operatorname{Ab}(\mathcal{C}, \mathcal{E}) \leq \operatorname{Ab}(\mathcal{C}, \mathcal{F}) \leq \operatorname{Ab}(\mathcal{D}, \mathcal{F})
$$

Proof. We may assume that $0 \leq \mathrm{Ab}(\mathcal{C}, \mathcal{F})=t<\infty$. Then $\operatorname{Ext}_{R}^{>t}(C, F)=0$ for any $C \in \mathcal{C}$ and $F \in \mathcal{F}$ with $\operatorname{Ext}_{R}^{\gg}(C, F)=0$, by the definition. In particular, since $\mathcal{E} \subseteq \mathcal{F}$, we have that $\operatorname{Ext}_{R}^{>t}(C, E)=0$ for any $C \in \mathcal{C}$ and $E \in \mathcal{E}$ with $\operatorname{Ext}_{R}^{\gg}(C, E)=0$. This shows that $\operatorname{Ab}(\mathcal{C}, \mathcal{E}) \leq t$. The remaining part is proved similarly. 
Let $\mathcal{C}, \mathcal{D}$ be two classes of $R$-modules. We have the following result.

\section{Lemma 2.6}

$$
\begin{aligned}
\operatorname{Ab}(\mathcal{C}, \mathcal{D}) & =\sup \{\operatorname{Ab}(M, \mathcal{D}) \mid M \in \mathcal{C}\} \\
& =\sup \{\operatorname{Ab}(\mathcal{C}, N) \mid N \in \mathcal{D}\} \\
& =\sup \{\operatorname{Ab}(C, D) \mid C \in \mathcal{C}, D \in \mathcal{D}\}
\end{aligned}
$$

Proof. By Proposition 2.5, we have that

$$
\begin{aligned}
\sup \{\operatorname{Ab}(C, D) \mid C \in \mathcal{C}, D \in \mathcal{D}\} & \leq \sup \{\operatorname{Ab}(M, \mathcal{D}) \mid M \in \mathcal{C}\} \\
& \leq \operatorname{Ab}(\mathcal{C}, \mathcal{D}) .
\end{aligned}
$$

To prove the other part, we may assume that $0 \leq \sup \{\operatorname{Ab}(C, D) \mid C \in \mathcal{C}$, $D \in \mathcal{D}\}=t<\infty$. Take any $C \in \mathcal{C}$ and $D \in \mathcal{D}$ with $\operatorname{Ext}_{R}^{\gg}(C, D)=0$, then we easily see that $\operatorname{Ext}_{R}^{>t}(C, D)=0$. Hence, we have that $\operatorname{Ab}(\mathcal{C}, \mathcal{D}) \leq t$.

We call $\operatorname{Ab}(\mathcal{C}, \mathcal{C})$ the global Auslander bound of the class $\mathcal{C}$. We denote by $\mathrm{GAb} R$ the global Auslander bound of $\operatorname{Mod} R$ and by gAb $R$ the global Auslander bound of $\bmod R$. Note that $\mathrm{gAb} R$ is just the Ext-index of $R$ in $[9,16]$.

If $R$ is an artin algebra, then there is a duality $\mathbf{D}$ between $\bmod R$ and $\bmod R^{o}$. Hence we can easily obtain that $\mathrm{gAb} R=\mathrm{gAb} R^{o}$ in this case.

To calculate the Auslander bound of a module, it is enough to calculate the Auslander bound of its syzygies, as the follow result shows.

Lemma 2.7 Let $M \in \operatorname{Mod} R$ and $\mathcal{C}$ be a class of $R$-modules.

(1) $0 \leq \mathrm{Ab}(M, \mathcal{C}) \leq m$ if and only if $\mathrm{Ab}\left(\Omega^{m} M, \mathcal{C}\right)=0$, where $\Omega^{m} M$ denotes an $m$-th syzygy of $M$.

(2) $0 \leq \mathrm{Ab}(\mathcal{C}, M) \leq m$ if and only if $\mathrm{Ab}\left(\mathcal{C}, \Omega^{-m} M\right)=0$, where $\Omega^{-m} M$ denotes an $m$-th cosyzygy of $M$.

Proof. (1) Since $M^{\gg}=\left(\Omega^{m} M\right)^{\gg}$ by Lemma $2.3(3)$ and $M^{>m}=\left(\Omega^{m} M\right)^{\geq 1}$ by dimension shifting, we see that

$$
\begin{aligned}
0 \leq \operatorname{Ab}(M, \mathcal{C}) \leq m & \Longleftrightarrow \varnothing \neq M^{\gg} \bigcap \mathcal{C} \subseteq M^{>m} \bigcap \mathcal{C} \\
& \Longleftrightarrow \varnothing \neq\left(\Omega^{m} M\right)^{\gg} \bigcap \mathcal{C} \subseteq\left(\Omega^{m} M\right)^{\geq 1} \bigcap \mathcal{C} \\
& \Longleftrightarrow \operatorname{Ab}\left(\Omega^{m} M, \mathcal{C}\right)=0 .
\end{aligned}
$$

The proof of (2) is dual to that of (1). 
By the above lemma, we easily obtain the following result.

Proposition 2.8 The following are equivalent for a class $\mathcal{C}$ such that $\operatorname{Ab}(\mathcal{C}, \mathcal{C}) \neq-1$

(1) The global Auslander bound of $\mathcal{C}$ is not more than $n$.

(2) $\operatorname{Ab}\left(\Omega^{n} \mathcal{C}, \mathcal{C}\right)=0$, where $\Omega^{n} \mathcal{C}$ denotes the class of all $n$-th syzygies of $R$-modules in $\mathcal{C}$.

(3) $\operatorname{Ab}\left(\mathcal{C}, \Omega^{-n} \mathcal{C}\right)=0$, where $\Omega^{-n} \mathcal{C}$ denotes the class of all $n$-th cosyzygies of $R$-modules in $\mathcal{C}$.

In some cases, the Auslander bound of a module can be tested by special modules, as the following theorem shows.

Theorem 2.9 Let $M \in \operatorname{Mod} R$.

(1) If $\mathrm{lAb} M<\infty$ and $R \in M^{\gg}$, then $\operatorname{lAb} M=\min \left\{t \mid R \in M^{>t}\right\}$.

(2) If $\mathrm{LAb} M<\infty$ and $R^{(\kappa)} \in M^{\gg}$ for all cardinals $\kappa$, then $\mathrm{LAb} M=$ $\min \left\{t \mid R^{(\kappa)} \in M^{>t}\right.$ for all cardinals $\left.\kappa\right\}$.

(3) If $\mathrm{RAb} M<\infty$ and $I \in \gg M$ for all injective $R$-modules $I$, then $\operatorname{RAb} M=\min \left\{t \mid I \in{ }^{>t} M\right.$ for all injective $R$-modules $\left.I\right\}$.

Proof. (1) Assume that $\operatorname{lAb} M=m<\infty$. Let $t=\min \left\{t \mid R \in M^{>t}\right\}$. Then $t<\infty$, since $R \in M^{\gg}$ by the assumption.

Note that $R \in \bmod R$, so we have that $R \in M^{>t} \bigcap \bmod R \subseteq M^{\gg} \bigcap \bmod R$. Now take any $N \in M^{\gg} \bigcap \bmod R$ and any projective resolution of $N: \cdots \rightarrow$ $P_{1} \rightarrow P_{0} \rightarrow N \rightarrow 0$ with each $P_{i}$ finitely generated projective. Then we have all $P_{i} \in M^{>t} \bigcap \bmod R$, and hence all $\Omega^{i} N \in M^{\gg} \bigcap \bmod R$, by Lemma 2.3 (1). Therefore, for all $i>t$, we obtain that

$$
\operatorname{Ext}_{R}^{i}(M, N) \simeq \operatorname{Ext}_{R}^{i+1}(M, \Omega N) \simeq \cdots \simeq \operatorname{Ext}_{R}^{i+m}\left(M, \Omega^{m} N\right)=0,
$$

by dimension shifting and the definition of $1 \mathrm{Ab} M$.

It follows that $N \in M^{>t} \bigcap \bmod R$. Consequently,

$$
M^{>t} \bigcap \bmod R=M^{\gg} \bigcap \bmod R,
$$

that is, $\mathrm{lAb} M \leq t$.

On the other hand, since $R \in M^{\gg} \bigcap \bmod R$ by assumptions, we have that $R \in M^{>m}$ by the definition of $1 \mathrm{Ab} M$. It follows that $t \leq m$ too. Hence the conclusion follows.

The proof of (2) is similar as (1) and the proof of (3) is dual to (2).

Immediately, we obtain the following corollary [16, Corollary 3.3]. 
Corollary 2.10 Assume that $\mathrm{id} R<\infty$.

(1) If $\operatorname{lAb} M<\infty$ for every $M \in \bmod R$, then $\operatorname{gAb} R=\mathrm{id} R$.

(2) If $R$ is left noetherian and $\mathrm{LAb} M<\infty$ for every $M \in \operatorname{Mod} R$, then $\mathrm{GAb} R=\mathrm{id} R$.

We note that assumptions in Theorem 2.9 (1) can not be removed. For example, let $R$ be an artin algebra of finite representation type with $\operatorname{id} R=\infty$. Then it is easy to see that $\mathrm{gAb} R<\infty$. However, it is obvious that there are modules $M \in \bmod R$ such that $\min \left\{t \mid R \in M^{>t}\right\}=\infty$. Thus the condition $R \in M^{\gg}$ is needed. Now let $R$ be a Gorenstein ring with $\mathrm{gAb} R=\infty$ (such rings exist by [11]). Then there are modules $M \in \bmod R$ such that $\mathrm{lAb} M=\infty$ by Corollary 2.10 (1). However, it is easy to see that $\min \left\{t \mid R \in M^{>t}\right\} \leq \operatorname{id} R<\infty$ for any $M \in \bmod R$. So the condition that $\operatorname{lAb} M<\infty$ is also needed.

We remark that it is an open question whether $\mathrm{GAb} R=\mathrm{gAb} R$ if $R$ is left noetherian.

The following theorem is our main result which relates Auslander bounds to Auslander-Reiten conjecture as claimed in the introduction.

Theorem 2.11 Let $M \in \operatorname{Mod} R$.

(1) Assume that $M \oplus R \in M^{\gg} \bigcap \bmod R$, then $\mathrm{lAb} M=\operatorname{pd} M$.

(2) Assume that $M \oplus R^{(\kappa)} \in M^{\gg}$ for any cardinal $\kappa$, then $\mathrm{LAb} M=\operatorname{pd} M$.

(3) Assume that $M, I \in{ }^{\gg} M$ for any injective $R$-module $I$, then $\mathrm{RAb} M=$ $\operatorname{id} M$.

Proof. (1) Clearly we need only prove that $\operatorname{pd} M \leq 1 \mathrm{Ab} M$.

We can assume that $1 \mathrm{Ab} M=m<\infty$. Since $M \in \bmod R$, we can take a projective resolution of $M: \cdots \rightarrow P_{1} \rightarrow P_{0} \rightarrow M \rightarrow 0$ with each $P_{i}$ finitely generated projective. Then each $\Omega^{i} M \in M^{\gg} \bigcap \bmod R$ by Lemma 2.3 (1), as $M \oplus R \in M^{\gg} \bigcap \bmod R$. It follows that $\Omega^{i} M \in M^{>m}$ for each $i$, by the definition of $1 \mathrm{Ab} M$. Now by applying the functor $\operatorname{Hom}_{R}\left(-, \Omega^{m+1} M\right)$ to the exact sequence $0 \rightarrow \Omega^{m} M \rightarrow P_{m-1} \rightarrow \cdots \rightarrow P_{0} \rightarrow M \rightarrow 0$, we obtain that $\operatorname{Ext}_{R}^{1}\left(\Omega^{m} M, \Omega^{m+1} M\right) \simeq \operatorname{Ext}_{R}^{2}\left(\Omega^{m-1} M, \Omega^{m+1} M\right) \simeq \cdots \simeq$ $\operatorname{Ext}_{R}^{m+1}\left(M, \Omega^{m+1} M\right)$ by dimension shifting. The latter is 0 since $\Omega^{m+1} M \in$ $M^{>m}$ by the above argument. It follows that the exact sequence $0 \rightarrow$ $\Omega^{m+1} M \rightarrow P_{m} \rightarrow \Omega^{m} M \rightarrow 0$ splits, and consequently, $\operatorname{pd} M \leq m$.

The proof of (2) is similar as (1) and the proof of (3) is dual to (2). 


\section{Homological conjectures}

As pointed out in [11, 17], Auslander's conjecture fails for artin algebras in general. However, we can consider a finitistic version of Auslander's conjecture. Let $R$ be a ring. We set

- $\operatorname{FLAb}(R):=\sup \{\operatorname{LAb} M \mid \operatorname{LAb} M<\infty\}$, and

- $\operatorname{flAb}(R):=\sup \{1 \mathrm{Ab} M \mid M \in \bmod R$ and $\operatorname{lAb} M<\infty\}$.

Similarly, we have notions $\operatorname{FRAb}(R)$ and $\operatorname{fr} \operatorname{Ab}(R)$ defined by right Auslander bounds. Note that $\mathrm{flAb}(R)=\operatorname{fr} \mathrm{Ab}\left(R^{o}\right)$ in case that $R$ is an artin algebra.

Now we formulate the following conjecture.

Finitistic Auslander Conjecture (FAC for short):

- (lFAC): $\operatorname{flAb}(R)<\infty$ for every artin algebra $R$, or dually

- (rFAC): $\operatorname{fr} \mathrm{Ab}(R)<\infty$ for every artin algebra $R$.

It is easy to see that the finitistic Auslander conjecture implies the finitistic dimension conjecture for artin algebras by Remark 2.2 (3).

It is also clear that

$$
\mathrm{flAb} R=\operatorname{fr} \mathrm{Ab} R<\infty \text { if } \mathrm{gAb} R<\infty,
$$

by Lemma 2.6. Similarly,

$$
\mathrm{FLAb} R=\mathrm{FRAb} R<\infty \text { if } \mathrm{GAb} R<\infty .
$$

For example, every group algebra $k G$ with $k$ a field and $G$ finite has the property $\operatorname{GAb}(k G)<\infty$, see [3, Theorem 2.4] and [4, Appendix A].

As the finitistic dimension conjecture fails for commutative noetherian rings in general, the conjecture (FAC) fails in the case, too. Moreover, it is pointed out in [4] that there is a commutative notherian ring $R$ with infinite Krull dimension such that $\mathrm{lAb} M<\infty$ for every $M \in \bmod R$ but $\mathrm{gAb} R=\infty$.

It is unknown whether gAbR $<\infty$ if $R$ is an artin algebra such that $\operatorname{lAb} M<\infty$ for every $M \in \bmod R$.

The following result gives a partial answer to the conjecture (FAC).

Proposition 3.1 Let $R$ be a ring.

(1) If $\operatorname{id} R<\infty$, then $\operatorname{flAb}(R)<\infty$.

(2) If $R$ is left noetherian and $\operatorname{id} R<\infty$, then $\operatorname{FLAb}(R)<\infty$.

Proof. (1) Indeed, we have that $\operatorname{lAb} M=\min \left\{t \mid R \in M^{>t}\right\} \leq \mathrm{id} R$ provided $1 \mathrm{Ab} M<\infty$ and $M \in \bmod R$, by Theorem 2.9 .

(2) If $R$ is left noetherian and $\operatorname{id} R<\infty$, then $\operatorname{id} R^{(\kappa)}<\infty$ for any cardinal $\kappa$. Now the conclusion follows from Theorem 2.9 again. 
Now we turn to other related homological conjectures.

Let $R$ be a ring and $T \in \operatorname{Mod} R$ with $S=\operatorname{End}_{R} T$. Recall from [18] that $T$ is Wakamatsu-tilting if it satisfies

(1) $T \in \bmod R$ and $T_{S} \in \bmod S^{o}$,

(2) $R \simeq \operatorname{End}\left(T_{S}\right)$, and

(3) $\operatorname{Ext}_{R}^{i}(T, T)=0=\operatorname{Ext}_{S^{o}}^{i}(T, T)$ for all $i>0$.

Equivalently, as shown in [18], $T$ is Wakamatsu-tilting if

(W1) $T \in \bmod R$,

(W2) $\operatorname{Ext}_{R}^{i}(T, T)=0$ for all $i>0$, and

(W3) there is an exact sequence $0 \rightarrow R \rightarrow{ }^{f_{0}} T_{0} \rightarrow^{f_{1}} T_{1} \rightarrow^{f_{2}} \ldots$ with each $T_{i} \in \operatorname{add} T$ and each $\operatorname{Im} f_{i} \in{ }^{>0} T$, for all $i \geq 0$.

It is clear that $T$ is Wakamatsu-tilting if and only if $T_{S}$ is Wakamatsutilting.

Recall also that $T$ is tilting $[2,15]$ if it satisfies

(T1) $T \in \bmod R$ and $\operatorname{pd} T<\infty$,

(T2) $\operatorname{Ext}_{R}^{i}(T, T)=0$ for all $i>0$, and

(T3) there is an exact sequence $0 \rightarrow R \rightarrow T_{0} \rightarrow \cdots \rightarrow T_{n} \rightarrow 0$ with each $T_{i} \in \operatorname{add} T$, for some integer $n$.

We note that $T$ is tilting if and only if $T_{S}$ is tilting, where $S=\operatorname{End}_{R} T$ [15]. The following conjecture is cited from [14].

Wakamatsu Tilting Conjecture (WTC for short): Every Wakamatsutilting module of finite projective dimension is tilting.

It is pointed out in [14] that, if the finitistic dimension conjecture holds for a ring $R$, then the conjecture (WTC) holds for $R$. We have an equivalent version of the conjecture (WTC) (and so we denote this conjecture by EWTC, where E means equivalent).

Proposition 3.2 The conjecture (WTC) holds for all rings if and only if the following conjecture (EWTC) holds for all rings $R$.

(EWTC): An $R$-module $T \in \bmod R$ is tilting if it satisfies conditions (T2) and (T3) in the definition of tilting modules. 
Proof. Let $R$ be a ring and $T \in \bmod R$ with $S=\operatorname{End}_{R} T$.

$($ EWTC $) \Rightarrow$ (WTC): Assume that $T$ is a Wakamatsu-tilting $R$-module with $\operatorname{pd} T<\infty$. Then we have an exact sequence $0 \rightarrow P_{n} \rightarrow \cdots \rightarrow P_{0} \rightarrow$ $T \rightarrow 0$ for some $n$. Applying the functor $\operatorname{Hom}_{R}(-, T)$, we obtain an induced exact sequence $0 \rightarrow S \rightarrow T_{0} \rightarrow \cdots \rightarrow T_{n} \rightarrow 0$ in $\bmod S^{o}$, since $\operatorname{Ext}_{R}^{i}(T, T)=0$ for all $i>0$. Since $T$ is a Wakamatsu-tilting $R$-module, $T$ is also a Wakamatsu-tilting $S^{o}$-module and so $\operatorname{Ext}_{S^{o}}^{i}(T, T)=0$ for all $i>0$. Hence we get that $T_{S}$ is tilting provided that (EWTC) holds for $S^{o}$. Consequently, $T$ is also a tilting $R$-module.

$(\mathrm{WTC}) \Rightarrow(\mathrm{EWTC})$ : If (T2) and (T3) in the definition of tilting modules hold for $T \in \bmod R$, then $T$ is Wakamatsu-tilting. Moreover, by applying the functor $\operatorname{Hom}_{R}(-, T)$ to the exact sequence in (T3), we easily see that $T_{S}$ is Wakamatsu-tilting with finite projective dimension. It follows that $T_{S}$ is tilting provided that (WTC) holds for $S^{o}$. Now by the left-right symmetry, we get that $T$ is tilting.

If we specify $n=0$ in the condition (T3), then $R \in \operatorname{add} T$. In this case, the conjecture (EWTC) is just the Auslander-Reiten conjecture.

The following result gives a partial answer to the conjecture (EWTC), which extends [4, Theorem A].

Proposition 3.3 Let $R$ be a ring. If $1 \mathrm{Ab} M<\infty$ for every $M \in \bmod R$, then the conjecture (EWTC) holds for $R$.

Proof. Assume that $T \in \bmod R$ satisfies conditions (T2) and (T3). Then we easily obtain that $T \oplus R \in T^{\gg}$. Now by the assumption and Theorem 2.11, we get that $\operatorname{pd} T=1 \mathrm{Ab} T<\infty$. It follows that $T$ is tilting.

We now consider another homological conjecture.

Gorenstein Symmetry Conjecture: Let $R$ be an artin algebra. Then $\operatorname{id} R<\infty$ if and only if $\operatorname{id}\left(R_{R}\right)<\infty$.

Gorenstein Symmetry conjecture clearly makes sense for any ring. It was proved in [4] that if $R$ is a two-sided noetherian ring such that $1 \mathrm{Ab} M<\infty$ for every $M \in \bmod R$ and $1 \mathrm{Ab} N<\infty$ for every $N \in \bmod R^{o}$, and (1) $R$ is an artin algebra, or (2) $R$ has a dualizing complex, then $\operatorname{id} R<\infty$ if and only if $\operatorname{id}_{R^{o}} R<\infty$ (whence, $\mathrm{id} R=\mathrm{id}_{R^{o}} R$ by [10]). The following result also gives a similar answer to the Gorenstein Symmetry conjecture. Note that we do not know if $\mathrm{LAb} M<\infty$ for every $M \in \operatorname{Mod} R$ provided that $1 \mathrm{Ab} M<\infty$ for every $M \in \bmod R$, even when $R$ is an artin algebra. We do not know whether $\operatorname{LAb} M<\infty$ for every $M \in \operatorname{Mod} R$ implies that $\mathrm{RAb} M<\infty$ for every $M \in \operatorname{Mod} R$. 
Proposition 3.4 Let $R$ be a two-sided noetherian ring. Assume that

(1) $\operatorname{LAb} M<\infty$ for every $M \in \operatorname{Mod} R$ and every $M \in \operatorname{Mod} R^{o}$, or

(2) $R$ has a dualizing complex and $\mathrm{rAb} M<\infty$ for every $M \in \bmod R$ and every $M \in \bmod R^{o}$.

Then $\operatorname{id} R<\infty$ if and only if $\operatorname{id} R^{o}<\infty$.

Proof. Assume (1) holds. If id $R<\infty$, then $\mathrm{GAb} R<\infty$ by assumptions and Corollary $2.10(2)$. It follows that $\operatorname{FRAb}(R)<\infty$. Using the fact that $\operatorname{RAb} M=\operatorname{id} M$ for any $R$-module $M$ with id $M<\infty$ in Remark 2.2 (3), we further obtain that the injective version of the finitistic dimension conjecture holds for $R$. This shows that id $R^{o}<\infty$ by [6, Proposition 7]. Similarly, we can prove that if $\operatorname{id} R^{o}<\infty$ then $\operatorname{id} R<\infty$.

Assume now (2) holds. If $\operatorname{id} R<\infty$, then for any $M \in \bmod R$ and any injective $R^{o}$-module $N$, it holds that

$$
\operatorname{Tor}_{(\mathrm{id} R)+1}^{R}(N, M) \simeq \operatorname{Hom}_{R^{o}}\left(\operatorname{Ext}_{R}^{(\mathrm{id} R)+1}(M, R), N\right)=0 .
$$

Hence $\operatorname{fd}_{R^{o}} N<\infty$. By the definition of dualizing complex [4, Section 3.4], $R^{o}$ has a dualizing complex if so is $R$. In this case, we have that all $R^{o}$-modules of finite flat dimension have finite projective dimension, by [12, Theorem]. It follows that $N \in{ }_{R^{o}} R$ for any injective $R^{o}$-module $N$. Now applying Theorem 2.11 to the $R^{o}$-module $R$, we obtain that $\operatorname{id}_{R^{o}} R=\operatorname{rAb} R$ and the latter is finite by assumptions. Thus, we have that $\operatorname{id} R^{o}<\infty$. The proof of the other part is also similar.

In the remaining part, we discuss a class of rings with finite global Auslander bound.

Let $R$ be a ring and $M$ an $R$-module. Assume that $n$ is a nonnegative integer. Following Goodearl and Zimmermann-Huisgen [7], we say that a projective resolution of $M$ is repetitive at degree $n$ if there exists a decomposition $\Omega^{n}(M)=P \oplus A_{i}$ such that $P$ is projective and each $A_{i}$ occurs as a direct summand of infinitely many $\Omega^{j}(M)$. The repetition index of $M$, denoted $\operatorname{rep}(M)$, is the least nonnegative integer $k$ such that there is a projective resolution of $M$ which is repetitive at degree $k$ (if such a $k$ exists), or $\infty$ (otherwise). The corresponding global repetition index is $\operatorname{Grep}(R)=\sup \{\operatorname{rep}(M) \mid M \in \operatorname{Mod} R\}$.

We have the following result which relates the repetition index to the Auslander bound.

Lemma 3.5 Let $R$ be a ring and $M \in \operatorname{Mod} R$. If $\operatorname{rep}(M)=m<\infty$, then $\mathrm{LAb} M \leq m$. 
Proof. Since $\operatorname{rep}(M)=m<\infty, M$ has a projective resolution such that $\Omega^{m}(M)=P \oplus A_{i}$ with $P$ projective and that each $A_{i}$ occurs as a direct summand of infinitely many $\Omega^{j}(M)$. So we have that $\Omega^{m} M \in \operatorname{add}(P \oplus$ $\left(\oplus_{i>t} \Omega^{i} M\right)$ ), for any $t$. Now take any $N \in M^{\gg}$ and assume that $N \in$ $M^{>t}$ for some $t$. It follows that $\operatorname{Ext}_{R}^{1}\left(\Omega^{m} M, N\right) \leq \operatorname{Ext}_{R}^{1}\left(\oplus_{i>t} \Omega^{i} M, N\right) \simeq$ $\prod_{i>t} \operatorname{Ext}_{R}^{i+1}(M, N)=0$ by dimension shifting and the definition of $M^{>t}$. Note that $\left(\Omega^{m} M\right)^{\gg}=M^{\gg}$ by Lemma 2.3, so $\operatorname{LAb}\left(\Omega^{m} M\right)=0$. It follows that $\mathrm{LAb} M \leq m$ by Lemma 2.7 .

Consequently, we obtain the following result.

Proposition 3.6 If $R$ is a ring with Grep $R<\infty$, then $\mathrm{GAb} R<\infty$. In this case, the conjecture (EWTC) holds. In particular, the Auslander-Reiten conjecture holds in this case.

Proof. By Lemma 3.5 and Proposition 3.3.

Let $R$ be an artin ring. Recall that $M \in \bmod R$ has a ultimately closed projective resolution if there is some $m$ such that the $m$-th syzygy $\Omega^{m} M \simeq \oplus M_{i}$ with each $M_{i} \in \operatorname{add}\left(\Omega^{m_{i}} M\right)$ for some $m_{i}<m$. In this case, we have that $\operatorname{rep}(M) \leq m$, see [7]. One defines artin rings such that every finitely generated module has an ultimately closed projective resolution to be of projective ultimately closed type. It was proved in [2] that the Auslander-Reiten conjecture holds for artin algebras of projective ultimately closed type. Lemma 3.5 and Proposition 3.3 together also imply that the conjecture (EWTC) and Auslander's conjecture hold for such artin algebras.

In [7], the authors studied finite dimensional algebra $R=\mathcal{O} / \pi \mathcal{O}$, where $\mathcal{O}$ is a classical order over a discrete valuation ring $D$ with uniformizing parameter $\pi$ and residue class field $K$. The homological properties of $\mathcal{O}$ are to a great extent determined by those of $R$ while the latter algebra is substantially easier to handle. In their paper, it was shown that, if $\operatorname{gd} \mathcal{O}=d<1$, then $\operatorname{Grep} R=d-1$, in particular, the finitistic dimension of $R$ is finite. Combining these with results in this paper and [4], we also know that in case $\mathcal{O}$ has finite global dimension, the algebra $\mathcal{O} / \pi \mathcal{O}$ also satisfies the Auslander conjecture, the conjecture (FAC), the Auslander-Reiten conjecture, Gorenstein Symmetry conjecture and the conjecture (EWTC).

Results in this section suggest the following conjecture which generalizes Auslander-Reiten conjecture.

Generalized Auslander-Reiten Conjecture: Let $R$ be a ring and $M \in$ $\bmod R$. If $M \oplus R \in M^{\gg}$, then $\operatorname{pd} M<\infty$.

By Lemma 3.5, the conjecture holds for artin algebras of projective ultimately closed type. 
Acknowledgements. It is a pleasure to thank the referee for his/her carefully reading and excellent suggestions.

\section{References}

[1] Angeleri-Hügel, L., Happel, D. and Krause, H.: Handbook of tilting theory. London Mathematical Society Lecture Note Series 332. Cambridge University Press, Cambridge, 2007.

[2] Auslander, M. and Reiten, I.: Applications of contravariantly finite subcategories. Adv. Math. 86 (1991), no. 1, 111-152.

[3] Benson, D. J., Carlson, J. F. and Robinson, G. R.: On the vanishing of group cohomology. J. Algebra 131 (1990), no. 1, 40-73.

[4] Christensen, L. W. And Holm, H.: Algebras that satisfy Auslander's condition on vanishing of cohomology. Math. Z. 265 (2010), no. 1, 21-40.

[5] Cowley, K. M.: One-sided bounds and the vanishing of Ext. J. Algebra 190 (1997), no. 2, 361-371.

[6] Fuller, K.R. And Wang, Y.: Redundancy in resolutions and finitistic dimensions of noetherian rings. Comm. Algebra 21 (1993), no. 8, 2983-2994.

[7] Goodearl, K. R. and Zimmermann-Huisgen, B.: Repetitive resolutions over classical orders and finite-dimensional algebras. In Algebras and modules II, 205-225. CMS Conf. Proc. 24. Amer. Math. Soc., Providence, RI, 1998.

[8] Happel, D.: Homological conjectures in representation theory of finitedimensional algebras. Sherbrook Lecture Notes Series, 1991. Available from www.math.ntnu.no/ oyvinso/Nordf jordeid/Program/references.html

[9] Huneke, C. And Jorgensoen, D. A.: Symmetry in the vanishing of Ext over Gorenstein rings. Math. Scand. 93 (2003), no. 2, 161-184.

[10] Iwanaga, Y.: On rings with finite self-injective dimension II. Tsukuba J. Math. 4 (1980), no. 1, 107-113.

[11] Jorgensen D. A. And SegA, L. M.: Nonvanishing cohomology and classes of Gorenstein rings. Adv. Math. 188 (2004), no. 2, 470-490.

[12] Jorgensen, P.: Finite flat and projective dimensions. Comm. Algebra 33 (2005), no. 7, 2275-2279.

[13] Kirkman, E. and Kuzmanovich, J.: On the finitistic dimension of fixed subrings. Comm. Algebra 22 (1994), no. 12, 4621-4635.

[14] Mantese, F. And Reiten, I.: Wakamatsu tilting modules. J. Algebra 278 (2004), no. 2, 532-552.

[15] Miyashita, Y.: Tilting modules of finite projective dimension. Math. Z. 193 (1986), no. 1, 113-146.

[16] Mori, I.: Symmetry in the vanishing of Ext over stably symmetric algebras. J. Algebra 310 (2007), no. 2, 708-729. 
[17] Smalø, S. O.: Local limitations of the Ext functor do not exist. Bull. London Math. Soc. 38 (2006), no. 1, 97-98.

[18] Wakamatsu, T.: On modules with trivial self-extensions. J. Algebra 114 (1988), no. 1, 106-114.

Recibido: 24 de noviembre de 2009

Revisado: 18 de diciembre de 2010

Jiaqun Wei

Institute of Mathematics

School of Mathematics Sciences

Nanjing Normal University

Nanjing 210046, China

weijiaqun@njnu.edu.cn

The author is supported by the National Natural Science Foundation of China (Grant No. 10971099). 